\title{
Quadrature on the half line and two-point Padé approximants to Stieltjes functions. Part III. The unbounded case*
}

\author{
A. Bultheel! C. Díaz-Mendoza ${ }^{\ddagger}$ P. González-Vera $\stackrel{\ddagger}{\ddagger}$ R. Orive
}

\begin{abstract}
Let $\alpha$ be a general, absolutely continuous measure, possibly complex, supported on $[0, \infty)$. Let $F_{\alpha}(z)$ denote its Cauchy transform. In this paper we prove, under suitable conditions, the convergence of two-point Padé type approximants to $F_{\alpha}$ and of the associated quadrature formulas $\sum_{j=1}^{n} A_{j} f\left(x_{j}\right)$ to the integral $\int_{0}^{\infty} f(x) d \alpha(x)$. These quadrature formulas can be of Gaussian or of interpolatory type. Estimates for the rate of convergence are also included.
\end{abstract}

Keywords: two-point Padé approximation, Stieltjes function, orthogonal Laurent polynomials, quadrature formula.

AMS Classification: 41A21, 30E05, 34E05, 41A55, 42C05.

\section{Introduction}

In this sequence of papers, we study quadrature formulas for a measure $\alpha$ on $[a, b]$ with $0 \leq a<b \leq \infty$. That is, we study approximants

$$
I_{n}(f)=\sum_{j=1}^{n} A_{j} f\left(x_{j}\right)
$$

for the integrals

$$
I_{\alpha}(f)=\int_{a}^{b} f(x) d \alpha(x)
$$

In part I [4], we assumed that $\alpha$ was a positive measure and we discussed the intimate relationship between these quadrature formulas and two-point Padé (2PA) and two-point Padé-type (2PTA) approximants for the corresponding Stieltjes function $F_{\alpha}$, which is the Cauchy transform of the measure $\alpha$ :

$$
F_{\alpha}(z)=\int_{a}^{b} \frac{d \alpha(x)}{x-z}=I_{\alpha}\left(\frac{1}{x-z}\right) .
$$

${ }^{*}$ This research was performed as part of the European project RoLLs under contract CHRX-CT93-0416.

${ }^{\dagger}$ Department of Computer Science, K.U.Leuven, Belgium

${ }_{\ddagger}^{\ddagger}$ Department Análisis Math., Univ. La Laguna, Tenerife, Spain 
The idea is quite simple since $I_{n}\left((x-z)^{-1}\right)$ is a rational function which approximates $F_{\alpha}(z)=$ $I_{\alpha}\left((x-z)^{-1}\right)$. These approximants satisfy a maximal number of interpolation conditions in 0 and $\infty$. The difference is that in a 2PA the freedom in numerator and denominator is fully used to satisfy as many interpolation conditions as possible, while in 2PTA, the denominator is fixed in advance, so that only the freedom in the numerator is used to interpolate as much as possible. Because of these interpolation conditions in 0 and $\infty$, the quadrature formulas will be exact in sets of Laurent polynomials $\Lambda_{-p, q}=\operatorname{span}\left\{z^{-p}, \ldots, z^{q}\right\}$ with $p+q$ as large as possible. For $2 \mathrm{PA}$, this maximal value is $p+q=2 n-1$, which means that these are Gaussian quadrature formulas, since this is the maximal domain of validity that can be obtained with $n$-point quadrature formulas. For 2PTA, we can only obtain $p+q=n-1$ and the quadrature formulas are of interpolatory type. The main result of part I was that the $2 \mathrm{PA}$ showed a monotonic convergence towards the Stieltjes function as $n$ increased.

In part II [3], we have studied convergence aspects. The convergence properties of the 2PA and 2PTA to $F_{\alpha}$ more or less reflects the convergence of the corresponding quadrature formulas.

At the same time we have introduced in part II, the fact that $\alpha$ can be a general, possibly complex, measure. Since for Gaussian quadrature formulas, the nodes $x_{j}$ should be chosen as the zeros of orthogonal Laurent polynomials with respect to $\alpha$, such a construction becomes impossible when $\alpha$ is not positive, in any case, it can not be guaranteed that their zeros are on the positive real line. Therefore we have to introduce in the general case an auxiliary positive Borel measure $\sigma$ on $[a, b]$ for which such orthogonal Laurent polynomials can be constructed. Taking the zeros of these Laurent polynomials allows to construct 2PTAs having such an orthogonal Laurent polynomial as their denominator and corresponding interpolatory quadrature formulas having the zeros of these orthogonal Laurent polynomials as their nodes.

Convergence is then proved for 2PA and Gaussian quadrature formulas as well as for 2PTA and interpolatory quadrature formulas which holds under certain technical conditions. Also estimates for the rate of convergence are given. Most of these results in part II were however obtained for an interval $[a, b]$ with $0 \leq a<b<\infty$ since the $b=\infty$ causes some extra complications. The case $b=\infty$ is the main concern of this paper.

The outline of the paper is as follows. In Section 2, we give the Markov approach to the construction of Gaussian quadrature formulas in the space of Laurent polynomials $\Lambda_{-p, q}$. This approach is based on Hermite interpolation of the integrand and it will provide some useful expressions for the error of the quadrature formulas. Expressions for the error of quadrature formulas of interpolatory type are given in Section 3. These are used in Section 4 to derive formulas for the error of $2 \mathrm{PA}$ and $2 \mathrm{PTA}$ to the Cauchy transform $F_{\alpha}$.

The actual convergence results are given in Section 5. Convergence results for 2PA were already given by López-Lagomasino, so the emphasis is on the convergence of 2PTA where it is assumed that $\alpha$ is really supported on the whole half line $[0, \infty)$. The corresponding convergence results for the quadrature formulas and estimates for the rate of convergence are also found in this section.

\section{Markov's approach to Gaussian quadrature in $\Lambda_{-p, q}$}

Let $\alpha$ be a distribution function on $[a, b], 0 \leq a \leq b \leq+\infty$. Let $p$ and $q$ be given integers such that $p \geq 0, q \geq-1$ and $p+q=2 n-1$ with $n$ a natural number. Let us consider the 
subspace of Laurent polynomials $\Lambda_{-p, q}=\operatorname{span}\left\{x^{j}: j=-p, \ldots, q\right\}$. We denote by $I_{\alpha}(f)$ the integral

$$
I_{\alpha}(f)=\int_{a}^{b} f(x) d x
$$

As we have already seen, there exist $n$ distinct nodes $x_{1}, \ldots, x_{n}$ in $(a, b)$ and positive weights $A_{1}, \ldots, A_{n}$ so that

$$
I_{\alpha}(f)=I_{n}(f):=\sum_{j=1}^{n} A_{j} f\left(x_{j}\right), \quad \forall f \in \Lambda_{-p, q} .
$$

Moreover, $\left\{x_{j}\right\}_{j=1}^{n}$ are the zeros of the $n$th orthogonal polynomial, say $Q_{n}$, with respect to $x^{-p} d \alpha(x)$ and $A_{j}=x_{j}^{p} C_{j}$ where $C_{j}$ are the Christoffel numbers for the distribution $\alpha$. This quadrature formula is the Gaussian quadrature formule for $\Lambda_{-p, q}$.

Let $F$ be a real function for which the derivative $F^{\prime}$ exists everywhere on $[0, \infty)$. Noting that $\operatorname{dim} \Lambda_{-p, q}=2 n$, we know [17] that for $n$ given distinct nodes $x_{j}$ in $(a, b)$ there exists a unique interpolating Laurent polynomial $H_{p, q}^{F}:=H_{p, q} \in \Lambda_{-p, q}$ such that

$$
H_{p, q}\left(x_{j}\right)=F\left(x_{j}\right) \quad \text { and } \quad H_{p, q}^{\prime}\left(x_{j}\right)=F^{\prime}\left(x_{j}\right), \quad j=1,2, \ldots, n .
$$

The construction of this interpolant is as follows. Set $\omega_{n}(x)=\prod_{j=1}^{n}\left(x-x_{j}\right)$ and define

$$
\ell_{j, n}(x)=\frac{x_{j}^{m(p)}}{x^{m(p)}} \frac{\omega_{n}(x)}{\left(x-x_{j}\right) \omega_{n}^{\prime}(x)}, \quad j=1, \ldots, n
$$

where $m(p)=p / 2$ if $p$ is even and $m(p)=(p-1) / 2$ if $p$ is odd; that is $m(p)=\lfloor p / 2\rfloor$. Clearly

$$
\ell_{j, n} \in \Lambda_{-m(p), n-1-m(p)} \subset \Lambda_{-p, q} \quad \text { and } \quad \ell_{j, n}^{2} \in \Lambda_{-p, 2 n-2-p} \subset \Lambda_{-p, q}
$$

(recall that $p+q=2 n-1$ ) and

$$
\ell_{j, n}\left(x_{k}\right)=\delta_{j, k}, \quad 1 \leq j, k \leq n
$$

where $\delta_{j, k}$ is the Kronecker delta.

For $j=1, \ldots, n$, define

$$
\begin{aligned}
& S_{j, n}(x)=\left[1-2 \alpha_{j, n}(x)\left(x-x_{j}\right) \ell_{j, n}^{\prime}(x)\right] \ell_{j, n}^{2}(x) \\
& T_{j, n}(x)=\alpha_{j, n}(x)\left(x-x_{j}\right) \ell_{j, n}^{2}(x)
\end{aligned}
$$

where $\alpha_{j, n}(x)=1$ if $p$ is even and $\alpha_{j, n}(x)=x_{j} / x$ if $p$ is odd. It is easily seen that both $S_{j, n}$ and $T_{j, n}$ belong to $\Lambda_{-p, q}$ and one has

$$
S_{j, n}\left(x_{k}\right)=\delta_{j, k} \quad \text { and } \quad T_{j, n}\left(x_{k}\right)=0, \quad 1 \leq j, k \leq n .
$$

Assume first that $p$ is odd, thus $\alpha_{j, n}(x)=x_{j} / x$. Then $\alpha_{j, n}(x)\left(x-x_{j}\right)=x_{j}-x_{j}^{2} / x$ and consequently

$$
S_{j, n}^{\prime}(x)=2 \ell_{j, n}(x) \ell_{j, n}^{\prime}(x)+2 \ell_{j, n}^{2} \ell_{j, n}^{\prime}\left(x_{j}\right) x_{j}^{2} / x^{2} .
$$

Thus $S_{j, n}\left(x_{k}\right)=0$ for $1 \leq j, k \leq n$. On the other hand,

$$
T_{j, n}(x)=\frac{x_{j}}{x}\left(x-x_{j}\right) \ell_{j, n}^{2}(x)
$$


so that $T_{j, n}\left(x_{k}\right)=0$ for $1 \leq j, k \leq n$. Taking the derivative gives

$$
T_{j, n}^{\prime}(x)=2 \ell_{j, n}(x) \ell_{j, n}^{\prime}(x)\left(x_{j}-\frac{x_{j}^{2}}{x}\right)+\frac{x_{j}^{2}}{x^{2}} \ell_{j, n}^{2}(x) .
$$

which implies $T_{j, n}^{\prime}\left(x_{k}\right)=\delta_{j, k}$ for $1 \leq j, k \leq n$.

The same conclusion can be achieved if $p$ is even, i.e. when $\alpha_{j, n}(x)=1$. Thus we may conclude that

Lemma 2.1 The unique Laurent polynomial $H_{p, q}^{F}=H_{p, q}$ from $\Lambda_{-p, q}$ which satisfies the interpolation conditions (2.2) is given by

$$
H_{p, q}(x)=\sum_{j=1}^{n} F\left(x_{j}\right) S_{j, n}(x)+\sum_{j=1}^{n} F^{\prime}\left(x_{j}\right) T_{j, n}(x),
$$

where $S_{j, n}$ and $T_{j, n}$ are given in (2.3)-(2.4).

As for the error we have

Lemma 2.2 Let $f$ be a real function for which $f^{(2 n)}$ exists everywhere in $[0, \infty)$ and let $F(x)=x^{-p} f(x)$. Then

$$
F(x)-H_{p, q}^{F}(x)=\frac{f^{(2 n)}(\xi) \omega_{n}^{2}(x)}{(2 n) ! x^{p}}, \quad \xi=\xi(x) \in(0, \infty)
$$

Proof. As in the polynomial case $(p=0)$ assume $x \notin\left\{x_{1}, \ldots, x_{n}\right\}$ and define

$$
V(t)=\left[F(t)-H_{p, q}^{F}(t)\right] t^{p}-K(x) \omega_{n}^{2}(t)
$$

where $K(x)=x^{p}\left[F(x)-H_{p, q}^{F}(x)\right] / \omega_{n}^{2}(x)$. Then $V(t)$ vanishes at $x, x_{1}, \ldots, x_{n}$. Since $t^{p} H_{p, q}^{F}(t)$ is a polynomial and $f(t)=t^{p} F(t)$, the derivative $V^{(2 n)}(t)$ exists in $(0, \infty)$. Hence by Rolle's Theorem, $V^{\prime}(t)$ has $n$ distinct zeros in $(0, \infty)$ which are different from $x, x_{1}, \ldots, x_{n}$. Also, by the interpolation conditions and since $\omega_{n}^{\prime}\left(x_{j}\right)=0$, one has $V^{\prime}\left(x_{j}\right)=0$ for $j=1, \ldots, n$. Thus $V^{\prime}(t)$ has $2 n$ distinct zeros in $(0, \infty)$. By applying Rolle's Theorem repeatedly, one deduces that $V^{(2 n)}(\xi)$ should vanish for some $\xi=\xi(x) \in(0, \infty)$. Since $x^{p} H_{p, q}^{F} \in \Pi_{2 n-1}$, it follows from (2.6) that

$$
V^{(2 n)}(\xi)=f^{(2 n)}(\xi)-(2 n) ! K(x)=0 .
$$

This concludes the proof.

Integration with respect to $d \alpha$ of this interpolation formula, provides a quadrature formula for $I_{\alpha}(F)$ involving values of the function $F$ and its derivatives.

$$
I_{\alpha}(F):=\int_{a}^{b} F(x) d \alpha(x)=\sum_{j=1}^{n} W_{j} F\left(x_{j}\right)+\sum_{j=1}^{n} \tilde{W}_{j} F^{\prime}\left(x_{j}\right)+E_{n}(F)
$$

where

$$
E_{n}(F)=\frac{1}{(2 n) !} \int_{a}^{b} \frac{f^{(2 n)}(\xi(x)) \omega_{n}^{2}(x)}{x^{p}} d \alpha(x)
$$


Let us now see what happens if we let $\left\{x_{j}\right\}_{j=1}^{n}$ be the zeros of the monic polynomial $P_{n}^{\alpha}$, orthogonal with respect to $x^{-p} d \alpha(x)$, i.e. we take the nodes such that $P_{n}^{\alpha}(x)=\omega_{n}(x)$. By the Mean Value Theorem, we get

$$
E_{n}(F)=\frac{f^{(2 n)}(\eta)}{(2 n) !} \int_{a}^{b}\left[P_{n}^{\alpha}(x)\right]^{2} x^{-p} d \alpha(x) .
$$

If we set $Q_{n}^{\alpha}(x)=\kappa_{n} P_{n}^{\alpha}(x)$ such that $Q_{n}^{\alpha}$ is orthonormal with respect to $x^{-p} d \alpha(x)$, i.e., $\kappa_{n}$ is the leading coefficient of the orthonormal polynomial, then

$$
E_{n}(F)=\frac{f^{(2 n)}(\eta)}{(2 n) ! \kappa_{n}^{2}}
$$

Clearly the quadrature formula (2.7) is exact in $\Lambda_{-p, q}$ since $F \in \Lambda_{-p, q}$, thus $F(x)=x^{-p} f(x)$ with $f \in \Pi_{2 n-1}$ and therefore $f^{(2 n)}(x) \equiv 0$.

Furthermore, with this choice of nodes, one has

$$
\begin{aligned}
\tilde{W}_{j} & =\int_{a}^{b} T_{j, n}(x) d \alpha(x)=\int_{a}^{b} \alpha_{j, n}(x)\left(x-x_{j}\right) \ell_{j, n}^{2}(x) d \alpha(x) \\
& =\int_{a}^{b} \alpha_{j, n}(x)\left(x-x_{j}\right)\left(\frac{x_{j}^{m(p)}}{x^{m(p)}} \frac{\omega_{n}(x)}{\left(x-x_{j}\right) \omega_{n}^{\prime}\left(x_{j}\right)}\right)^{2} d \alpha(x) \\
& =C_{j} \int_{a}^{b} \alpha_{j, n}(x) P_{n}^{\alpha}(x) \frac{P_{n}^{\alpha}(x)}{\left(x-x_{j}\right)} \frac{d \alpha(x)}{x^{2 m(p)}}
\end{aligned}
$$

If $p$ is even, $\alpha_{j, n}(x)=1, m(p)=p / 2$. Thus the previous integral is zero because $P_{n}^{\alpha}$ is orthogonal to all polynomials of degree less than $n$ with respect to $x^{-p} d \alpha(x)$. Similarly, if $p$ is odd, $\alpha_{j, n}(x)=x_{j} / x$ and $m(p)=(p-1) / 2$, so that by similar arguments, we find that the integral is also zero for $p$ odd. Thus all the $\tilde{W}_{j}$ are zero and therefore the quadrature formula is of the form

$$
I_{n}(F)=\sum_{j=1}^{n} W_{j} F\left(x_{j}\right)
$$

Since it is exact in $\Lambda_{-p, q}$ we conclude by virtue of [4, Theorem 3.1], that this formula coincides with the corresponding Gaussian quadrature formula in $\Lambda_{-p, q}$. Thus the weights $W_{j}$ of (2.8) and the weights $A_{j}$ of (2.1) are the same. That is, it should hold that $W_{j}=A_{j}$ for $j=1, \ldots, n$. There is another, direct way to see this. Because both quadrature formulas are exact in $\Lambda_{-p, q}$, we find that

$$
\begin{aligned}
\int_{a}^{b} H_{p, q}^{F}(x) d \alpha(x) & =\sum_{j=1}^{n} A_{j} H_{p, q}^{F}\left(x_{j}\right)=\sum_{j=1}^{n} A_{j} F\left(x_{j}\right) \\
& =\sum_{j=1}^{n} W_{j} H_{p, q}^{F}\left(x_{j}\right)=\sum_{j=1}^{n} W_{j} F\left(x_{j}\right)
\end{aligned}
$$

so that

$$
\sum_{j=1}^{n} A_{j} F\left(x_{j}\right)=\sum_{j=1}^{n} W_{j} F\left(x_{j}\right)
$$

Taking $F(x)=\ell_{j, n}(x)$ and using $\ell_{j, n}\left(x_{k}\right)=\delta_{j, k}$, it follows that $W_{j}=A_{j}$ for $j=1, \ldots, n$. Thus we have proved the following theorem 
Theorem 2.3 Let $Q_{n}^{\alpha}$ denote the $n$th orthonormal polynomial with respect to the distribution $x^{-p} d \alpha(x), p \geq 0$ on $[a, b]$ with $0 \leq a<b \leq \infty$. Let $\kappa_{n}>0$ be the leading coefficient of $Q_{n}^{\alpha}$. Let $\left\{x_{j}\right\}_{j=1}^{n}$ and $\left\{A_{j}\right\}_{j=1}^{n}$ be the nodes and weights of the n-point Gaussian formula in $\Lambda_{-p, q}$ where $0 \leq p \leq 2 n, q \geq-1$, and $p+q=2 n-1$. Let $f(t)$ be a real function for which $f^{(2 n)}(t)$ exists for $a \leq t \leq b$ where $0 \leq a<b \leq \infty$ and let $F(t)=t^{-p} f(t)$. Then

$$
I_{\alpha}(F):=\int_{a}^{b} F(x) d \alpha(x)=I_{n}(F)+E_{n}(F)
$$

where

$$
I_{n}(F)=\sum_{j=1}^{n} A_{j} F\left(x_{j}\right) \quad \text { and } \quad E_{n}(F)=\frac{f^{(2 n)}(\eta)}{(2 n) ! \kappa_{n}^{2}}, \quad \eta \in(a, b) .
$$

For the computation of the weights $A_{j}$, we can use several expressions. First, we know that $A_{j}=x_{j}^{p} C_{j}$, for $j=1, \ldots, n$ where the $C_{j}$ are the Christoffel numbers for $x^{-p} d \alpha(x)$. Hence

$$
A_{j}=\frac{x_{j}^{p}}{\omega_{n}^{\prime}\left(x_{j}\right)^{2}} \int_{a}^{b} \frac{\omega_{n}^{2}(x)}{\left(x-x_{j}\right)^{2}} \frac{d \alpha(x)}{x^{p}}, \quad j=1, \ldots, n .
$$

On the other hand

$$
A_{j}=\int_{a}^{b} S_{j, n}(x) d \alpha(x)
$$

However, the latter expression can be simplified considerably since

$$
A_{j}=\int_{a}^{b} \ell_{j, n}^{2}(x) d \alpha(x)=\int_{a}^{b} \ell_{j, n}(x) d \alpha(x) .
$$

Indeed, for the first equality, take into account that $\ell_{j, n} \in \Lambda_{-p, q}$ and $\ell_{j, n}\left(x_{k}\right)=\delta_{j, k}$ and hence

$$
\int_{a}^{b} \ell_{j, n}^{2}(x) d \alpha(x)=\sum_{k=1}^{n} A_{k} \ell_{j, n}^{2}\left(x_{k}\right)=A_{j}, \quad j=1, \ldots, n
$$

For the next simplification, we should show that $\int_{a}^{b}\left[\ell_{j, n}^{2}(x)-\ell_{j, n}(x)\right] d \alpha(x)$ is zero. This integral is

$$
\int_{a}^{b} \omega_{n}(x)\left[\frac{x_{j}^{m(p)} \omega_{n}(x)-x^{m(p)}\left(x-x_{j}\right) \omega_{n}^{\prime}\left(x_{j}\right)}{\left(x-x_{j}\right)^{2}}\right] \frac{d \alpha(x)}{x^{2 m(p)}}=\int_{a}^{b} \omega_{n}(x) \frac{T(x)}{\left(x-x_{j}\right)^{2}} \frac{d \alpha(x)}{x^{2 m(p)}} .
$$

If $0 \leq p \leq 2 n$, then $T(x)$ is a polynomial of degree at most $n$ when $p$ is even as well as when $p$ is odd. Moreover $T\left(x_{j}\right)=T^{\prime}\left(x_{j}\right)=0$ so that $S(x)=\left(x-x_{j}\right)^{-2} T(x) \in \Pi_{n-2}$. If $p$ is even then $2 m(p)=p$ and $(2.12)$ becomes

$$
\int_{a}^{b} \omega_{n}(x) S(x) \frac{d \alpha(x)}{x^{p}}=0
$$

because $\omega_{n} \perp \Pi_{n-1}$ with respect to $x^{-p} d \alpha(x)$. If $p$ is odd, (2.12) becomes

$$
\int_{a}^{b} \omega_{n}(x) x S(x) \frac{d \alpha(x)}{x^{p}}
$$

which is also zero because $x S(x) \in \Pi_{n-1}$. Now the second equality in (2.11) has been proved. 
Note also that (2.10) and the first integral of (2.11) are the same when $p$ is even. For $p$ odd, the first integral of (2.11) gives the same expression as in (2.10) but with $p$ replaced by $p-1$. However, using the orthogonality property of $\omega_{n}$ with respect to $x^{-p} d \alpha(x)$ one can show that both expressions are the same.

Let us now assume that a sequence $\{p(n)\}$ of nonnegative integers is given such that $0 \leq p(n) \leq n$ and $p(n-1) \leq p(n)$. Set $q(n)=n-1-p(n)$. For each $n \geq 0$, set $($ see $[4,3])$

$$
\mathcal{R}_{n}=\Lambda_{-p(n+1), q(n+1)} .
$$

Thus $\operatorname{dim} \mathcal{R}_{n}=n+1$ and $\mathcal{R}_{n} \subset \mathcal{R}_{n+1}$. Let $\left\{R_{j}\right\}_{j=0}^{n}$ be an orthogonal basis for $\mathcal{R}_{n}, n=$ $0,1, \ldots$. We know that $R_{n}$ has $n$ distinct zeros $\left\{x_{j}\right\}_{j=1}^{n}$ on $(a, b)$. If we assume that $p(n)+$ $p(n+1)=p(2 n)$, then the zeros $\left\{x_{j}\right\}_{j=1}^{n}$ of $R_{n}$ are the nodes in the Gaussian quadrature formula for

$$
\Lambda_{-(p(n)+p(n+1)), q(n)+q(n+1)}=\Lambda_{-p(2 n), q(2 n)}=\mathcal{R}_{2 n-1} .
$$

By Theorem 2.3, one can write

$$
\begin{aligned}
E_{n}(F) & :=I_{\alpha}(F)-I_{n}(F)=\frac{f^{(2 n)}(\eta)}{(2 n) !} \int_{a}^{b}\left[Q_{n}^{\alpha}(x)\right]^{2} \frac{d \alpha(x)}{x^{p(2 n)}} \\
& =\frac{f^{(2 n)}(\eta)}{(2 n) !} \int_{a}^{b} \frac{\left[Q_{n}^{\alpha}(x)\right]^{2}}{x^{p(n)+p(n+1)}} \frac{x^{p(n+1)}}{x^{p(n+1)}} d \alpha(x) \\
& =\frac{f^{(2 n)}(\eta)}{(2 n) !} \int_{a}^{b}\left[\frac{Q_{n}^{\alpha}(x)}{x^{p(n+1)}}\right]^{2} x^{p(n+1)-p(n)} d \alpha(x) .
\end{aligned}
$$

Setting $\lambda(n)=p(n+1)-p(n)$ and because $R_{n}(x)=x^{-p(n)} Q_{n}^{\alpha}(x)$ is an orthogonal basis for $\mathcal{R}_{n}$, one has

$$
E_{n}(F)=\frac{f^{(2 n)}(\eta)}{(2 n) !} \int_{a}^{b} R_{n}^{2}(x) x^{\lambda(n)} d \alpha(x) .
$$

If we set $\tilde{R}_{n}=R_{n} \kappa_{n}$ such that $\tilde{R}_{n}$ is orthonormal, it results that

$$
E_{n}(F)=\frac{f^{(2 n)}(\eta) \nu(n)}{(2 n) ! \kappa_{n}^{2}}, \quad \text { where } \quad \nu(n)=\int_{a}^{b} \tilde{R}_{n}^{2}(x) x^{\lambda(n)} d \alpha(x)
$$

If we take $p(n)=\lfloor n / 2\rfloor,(\lfloor x\rfloor$ is the integer part of $x)$ then it clearly satisfies $p(n)+p(n+1)=$ $p(2 n)$. This choice coincides with the sequence of subspaces of Laurent polynomials studied by Jones et al. [9]. Now $\lambda(n)=0$ if $n$ is even and 1 if $n$ is odd. Therefore

$$
\nu(n)= \begin{cases}1 & \text { if } n \text { is even } \\ \int_{a}^{b} x \tilde{R}_{n}^{2}(x) d \alpha(x) & \text { if } n \text { is odd }\end{cases}
$$

(See [9, Theorem 3.1].)

Remark 2.1 If $n$ is odd, then integrating by parts, we find

$$
\begin{aligned}
\nu(n) & =\left.x \int_{a}^{x} \tilde{R}_{n}^{2}(t) d \alpha(t)\right|_{a} ^{b}-\int_{a}^{b}\left[\int_{a}^{x} \tilde{R}_{n}^{2}(t) d \alpha(t)\right] d x \\
& =b-\int_{a}^{b}\left[\int_{a}^{x} \tilde{R}_{n}^{2}(t) d \alpha(t)\right] d x<b .
\end{aligned}
$$




\section{Error for quadrature formulas of interpolating type}

Take as before integers $p$ and $q$ such that $p \geq 0, q \geq-1$ and $p+q=n-1$. For $n$ distinct nodes $\left\{x_{j}\right\}_{j=1}^{n}\left(x_{i} \neq 0\right)$ we know that (note $\left.\operatorname{dim} \Lambda_{-p, q}=n\right)$ there exist $n$ weights $A_{1}, \ldots, A_{n}$ uniquely defined such that

$$
\tilde{I}_{n}(F):=\sum_{j=1}^{n} \tilde{A}_{j} F\left(x_{j}\right)=I_{\alpha}(F):=\int_{a}^{b} F(x) d \alpha(x), \quad \forall F \in \Lambda_{-p, q} .
$$

If $\tilde{H}_{p, q}^{F}(x)$ denotes the Laurent polynomial in $\Lambda_{-p, q}$ interpolating $F$ at the nodes $\left\{x_{j}\right\}_{j=1}^{n}$, one has

$$
\tilde{I}_{n}(F)=\sum_{j=1}^{n} \tilde{A}_{j} F\left(x_{j}\right)=I_{\alpha}\left(\tilde{H}_{p, q}^{F}\right) .
$$

If we define

$$
L_{j, n}(x)=\frac{x_{j}^{p}}{x^{p}} \frac{\omega_{n}(x)}{\left(x-x_{j}\right) \omega_{n}^{\prime}\left(x_{j}\right)}, \quad \omega_{n}(x)=\prod_{j=1}^{n}\left(x-x_{j}\right),
$$

one sees that

$$
L_{j, n} \in \Lambda_{-p, n-1-p}=\Lambda_{-p, q} \quad \text { and } \quad L_{j, n}\left(x_{k}\right)=\delta_{j, k}
$$

Thus, we can write

$$
\tilde{H}_{p, q}^{F}(x)=\sum_{j=1}^{n} L_{j, n}(x) F\left(x_{j}\right) \quad \text { and } \quad \tilde{A}_{j}=I_{\alpha}\left(L_{j, n}\right), \quad j=1, \ldots, n .
$$

Let $f(t)$ be a real function for which $f^{(n)}$ exists in $[a, b](0 \leq a<b \leq \infty)$ and let $F(t)=$ $t^{-p} f(t)$. Take $x \notin\left\{x_{1}, \ldots, x_{n}\right\}$, and define for all $t \in(a, b)$

$$
V(t)=\left[F(t)-\tilde{H}_{p, q}^{F}(t)\right] t^{p}-K(x) \omega_{n}(t)
$$

where

$$
K(x)=\frac{\left[F(x)-\tilde{H}_{p, q}^{F}(x)\right] x^{p}}{\omega_{n}(x)} .
$$

Then $V(t)$ vanishes at $x, x_{1}, \ldots, x_{n}$. Since $t^{p} \tilde{H}_{p, q}^{F}(t)$ is a polynomial and $f(t)=t^{p} F(t)$, the derivative $V^{(n)}(t)$ will exist for all $t \in(a, b)$. Like in the Gaussian case, it follows by repeated application of Rolle's Theorem that there exists a $\xi=\xi(x) \in(a, b)$ such that $V^{(n)}(\xi)=0$ and hence

$$
V^{(n)}(\xi)=f^{(n)}(\xi)-n ! K(x)=0
$$

so that

$$
F(x)-\tilde{H}_{p, q}^{F}(x)=\frac{f^{(n)}(\xi) \omega_{n}(x)}{n ! x^{p}}
$$

Hence we get

$$
\tilde{E}_{n}(F):=I_{\alpha}(F)-\tilde{I}_{n}(F)=I_{\alpha}\left(F-\tilde{H}_{p, q}^{F}\right)=\frac{1}{n !} \int_{a}^{b} f^{(n)}(\xi(x)) \omega_{n}(x) x^{-p} d \alpha(x) .
$$

Note that this is also true when $\alpha$ is a more general distribution, e.g. complex. Now consider an auxiliary positive Borel measure $\sigma$ such that $d \sigma(x)=\sigma^{\prime}(x) d x$, with $\sigma^{\prime}>0$ a.e. on $[a, b]$. 
Let $N$ be a fixed nonnegative integer and let $P_{n}^{\sigma}(x)$ be the $n$th monic orthogonal polynomial with respect to $x^{-N} d \sigma(x)$. The nodes $\left\{x_{j}\right\}_{j=1}^{n}$ to be taken are the zeros of $P_{n}^{\sigma}(x)$, thus

$$
P_{n}^{\sigma}(x)=\omega_{n}(x)=\left(x-x_{1}\right) \cdots\left(x-x_{n}\right), \quad x_{j} \in[a, b], j=1, \ldots, n .
$$

Assume in addition that also $\alpha$ is absolutely continuous: $d \alpha(x)=\alpha^{\prime}(x) d x$ and that there exists a positive constant $K_{1}$ such that

$$
\int_{a}^{b} \frac{\left|\alpha^{\prime}(x)\right|^{2}}{\sigma^{\prime}(x)} d x=K_{1}^{2}<\infty
$$

Using the Cauchy-Schwarz inequality, one can write

$$
\begin{aligned}
\left|\tilde{E}_{n}(F)\right| & =\frac{1}{n !}\left|\int_{a}^{b} f^{(n)}(\xi(x)) P_{n}^{\sigma}(x) x^{-p} d \alpha(x)\right| \\
& =\frac{1}{n !}\left|\int_{a}^{b} f^{(n)}(\xi(x)) P_{n}^{\sigma}(x) \frac{\alpha^{\prime}(x)}{x^{p}} \frac{\sqrt{\sigma^{\prime}(x)}}{\sqrt{\sigma^{\prime}(x)}} \frac{x^{N / 2}}{x^{N / 2}} d x\right| \\
& \leq \frac{1}{n !}\left|\int_{a}^{b}\left(f^{(n)}(\xi(x))\right)^{2}\left[P_{n}^{\sigma}(x)\right]^{2} \frac{\sigma^{\prime}(x)}{x^{N}} d x\right|^{1 / 2}\left|\int_{a}^{b} \frac{\left|\alpha^{\prime}(x)\right|^{2}}{\sigma^{\prime}(x)} x^{N-2 p} d x\right|^{1 / 2} .
\end{aligned}
$$

Setting $N=2 p$, one obtains

$$
\left|\tilde{E}_{n}(F)\right| \leq \frac{K_{1}}{n !}\left|\int_{a}^{b}\left(f^{(n)}(\xi(x))\right)^{2}\left[P_{n}^{\sigma}(x)\right]^{2} \frac{\sigma^{\prime}(x)}{x^{2 p}} d x\right|^{1 / 2} .
$$

Now if $Q_{n}^{\sigma}(x)=\gamma_{n} P_{n}^{\sigma}(x), \gamma_{n}>0$ is the orthonormal version of $P_{n}^{\sigma}$, i.e. $Q_{n}^{\sigma}$ is orthonormal with respect to $x^{-2 p} d \sigma(x)$, and if we use the Mean Value Theorem, we get

$$
\left|\tilde{E}_{n}(F)\right| \leq \frac{K_{1}\left|f^{(n)}(\eta)\right|}{n ! \gamma_{n}}, \quad \eta \in(a, b)
$$

Thus the following theorem has been proved.

Theorem 3.1 Let $\alpha$ be a general (possibly complex) measure such that $\int_{a}^{b} d|\alpha|<\infty$ and $\operatorname{supp}(\alpha) \subset[a, b]$ with $0 \leq a<b \leq \infty$. Let $\sigma$ be a distribution function on $[a, b]$ with $\sigma^{\prime}>0$ a.e. Assume that $d \alpha(x)=\alpha^{\prime}(x) d x$ and that there exists a positive constant $K_{1}$ such that

$$
\int_{a}^{b} \frac{\left|\alpha^{\prime}(x)\right|^{2}}{\sigma^{\prime}(x)} d x=K_{1}^{2}<\infty
$$

Let $Q_{n}^{\sigma}$ denote the $n$th orthonormal polynomial with respect to $x^{-2 p} d \sigma(x),(p \geq 0)$ with leading coefficient $\gamma_{n} \neq 0$. Let $\tilde{I}_{n}(f)=\sum_{j=1}^{n} \tilde{A}_{j} f\left(x_{j}\right)$ be the interpolatory quadrature formula in $\Lambda_{-p, n-1-p}$ with interpolation points the zeros of $Q_{n}^{\sigma}$. Let $f(t)$ be a real function for which $f^{(n)}(t)$ exists for all $t \in(a, b)$ and let $F(t)=t^{-p} f(t)$. Then

$$
\left|\tilde{E}_{n}(F)\right|:=\left|I_{\alpha}(F)-\tilde{I}_{n}(F)\right| \leq \frac{K_{1}\left|f^{(n)}(\eta)\right|}{n !\left|\gamma_{n}\right|}, \quad \eta \in(a, b) .
$$




\section{Error bounds for two-point Padé and Padé-type ap- proximants of Cauchy transforms}

Let us first consider the Cauchy transform of the distribution $\alpha$, i.e.

$$
F_{\alpha}(z)=\int_{a}^{b} \frac{d \alpha(x)}{z-x}=I_{\alpha}\left(\frac{1}{z-x}\right), \quad z \in \hat{\mathbb{C}}-[a, b] .
$$

$F_{\alpha}(z)$ admits the asymptotic expansions

$$
\begin{aligned}
L^{0}(z) & =-\sum_{j=0}^{\infty} c_{-(j+1)} z^{j}, \quad z \rightarrow 0 \\
L^{\infty}(z) & =\sum_{j=1}^{\infty} c_{j-1} z^{-j}, \quad z \rightarrow \infty .
\end{aligned}
$$

Take the integer $p$ such that $0 \leq p \leq 2 n$ and consider the Gaussian quadrature formula in $\Lambda_{-p, 2 n-1-p}=\Lambda_{-p, q}(p \geq 0, q=2 n-1-p \geq-1)$ :

$$
I_{n}(f)=\sum_{j=1}^{n} A_{j} f\left(x_{j}\right)
$$

and set

$$
F_{n}(z)=I_{n}\left(\frac{1}{z-x}\right)=\sum_{j=1}^{n} \frac{A_{j}}{z-x_{j}} .
$$

This $F_{n}$ is a rational function of type $(n-1, n)$ satisfying

$$
\begin{aligned}
L^{0}(z)-F_{n}(z) & =O\left(z^{p}\right), \quad z \rightarrow 0 \\
L^{\infty}(z)-F_{n}(z) & =O\left(z^{-(q+2)}\right), \quad z \rightarrow \infty .
\end{aligned}
$$

This says that $F_{n}(z)=[p / n]_{F_{\alpha}}(z)$ is the two-point Padé approximant $(2 \mathrm{PA})$ to $F_{\alpha}(z)$ or equivalently to the pair $\left(L^{0}, L^{\infty}\right)$. See $[6]$. Set

$$
R_{n}(z):=F_{\alpha}(z)-F_{n}(z)=E_{n}\left(\frac{1}{z-x}\right), \quad z \notin[a, b]
$$

Consider $f(x)=z^{p} /(z-x) \in C^{\infty}([a, b])$, so that by formula (2.9) one has

$$
R_{n}(z)=\frac{f^{(2 n)}(\eta)}{(2 n) ! \kappa_{n}^{2}} \quad \text { for some } \eta \in(a, b)
$$

But, since $f^{(2 n)}(x)=(2 n) !(z-x)^{-(2 n+1)} z^{p}$, one gets

$$
R_{n}(z)=\frac{z^{p}}{\kappa_{n}^{2}(z-\eta)^{2 n+1}}, \quad 0 \leq p \leq 2 n, \quad \eta \in(a, b) .
$$

For $z \in \mathbb{R}-[a, b]$, one has

$$
\begin{aligned}
\left|R_{n}(z)\right| & \leq \frac{|z|^{p}}{\kappa_{n}^{2}|z-b|^{2 n+1}} \quad \text { if } z>b \quad(b \text { finite }) \\
& \leq \frac{|z|^{p}}{\kappa_{n}^{2}|z-a|^{2 n+1}} \quad \text { if } z<a
\end{aligned}
$$


Remark 4.1 Clearly, when $b=\infty$, only the second inequality is meaningful. Also note that (4.1)-(4.2) coincide with the formulas given by Jones et al. in [9, Theorem 3.2] when taking $p=p(n)=n$.

Let us next assume that $\alpha$ is a complex distribution and let us consider an $n$-point interpolatory quadrature formula $\tilde{I}_{n}$ in $\Lambda_{-p, q}$ where $p$ and $q$ are integers such that $p \geq 0$, $q \geq-1, p+q=n-1$. Set $\tilde{I}_{n}(f)=\sum_{j=1}^{n} A_{j} f\left(x_{j}\right)$ where $\left\{x_{j}\right\}$ are the zeros of $Q_{n}^{\sigma}(x)$, the $n$th orthonormal polynomial with respect to the distribution $x^{-2 p} d \sigma(x)$. Moreover set

$$
\tilde{I}_{n}\left(\frac{1}{z-x}\right)=\sum_{j=1}^{n} \frac{\tilde{A}_{j}}{z-x_{j}}=: \tilde{F}_{n}(z)
$$

such that

$$
L^{0}(z)-\tilde{F}_{n}(z)=O\left(z^{p}\right), \quad z \rightarrow 0 \quad \text { and } \quad L^{\infty}(z)-\tilde{F}_{n}(z)=O\left(z^{-(q+1)}\right), \quad z \rightarrow \infty .
$$

This rational function $\tilde{F}_{n}$ of type $(n-1, n)$ represents a $(p / n)_{F_{\alpha}}$, that is the two-point Padé-type approximant (2PTA) to $F_{\alpha}(z)$, or equivalently to the pair $\left(L^{0}, L^{\infty}\right)$. See [3].

Under the same hypothesis as in Theorem 3.1, one now has for the error $\tilde{R}_{n}=F_{\alpha}-\tilde{F}_{n}$ :

$$
\left|\tilde{R}_{n}(z)\right|=\left|\left(I_{\alpha}-\tilde{I}_{n}\right)\left(\frac{1}{z-x}\right)\right|=\left|\tilde{E}_{n}\left(\frac{1}{z-x}\right)\right| \leq \frac{K_{1}|z|^{p}}{\left|\gamma_{n}\right||z-\eta|^{n+1}}, \quad \eta \in(a, b)
$$

Thus

$$
\begin{aligned}
\left|\tilde{R}_{n}(z)\right| & \leq \frac{K_{1}|z|^{p}}{\left|\gamma_{n}\right||z-b|^{n+1}}, \quad \text { if } z>b, \quad(b \text { finite }) \\
& \leq \frac{K_{1}|z|^{p}}{\left|\gamma_{n}\right||z-a|^{n+1}}, \quad \text { if } z<a
\end{aligned}
$$

\section{Convergence of 2PA and 2PTA}

So far, we have assumed that $\alpha$ is a finite Borel measure supported on $[a, b]$ with $0 \leq a<$ $b \leq \infty$.

In [3], results about the convergence of $2 \mathrm{PA}$ and 2PTA to the Cauchy transform

$$
F_{\alpha}(x)=\int_{a}^{b} \frac{d \alpha(x)}{z-x}
$$

of the measure were given under the restriction $b<\infty$. We have seen that for this case, Carleman type conditions were not required. When $0<a<b<\infty$, we also gave estimates in that paper for the rates of convergence of the 2PA and 2PTA and of the corresponding quadrature formulas of Gaussian and interpolatory type. Note that the case $[a, \infty)$ with $a>0$ reduces to the case $[0, b]$ with $b<\infty$ by a change of variables $t=1 / x$ which maps the interval $[a, \infty)$ to $[0,1 / a]$ when $a>0$. As for the corresponding Cauchy transform, one has with $z=1 / u$

$$
F_{\alpha}(z)=\int_{a}^{\infty} \frac{d \alpha(x)}{z-x}=\int_{0}^{1 / a} \frac{d \alpha(1 / t)}{u^{-1}-t^{-1}}=-u \int_{0}^{1 / a} \frac{d \tilde{\alpha}(t)}{u-t}
$$

with $d \tilde{\alpha}(t)=t d \alpha\left(t^{-1}\right)$. 
Thus, we shall in this section assume that $\alpha$ is actually supported on the half line $[0, \infty)$. We assume that all the moments

$$
c_{k}=\int_{0}^{\infty} x^{k} d \alpha(x), \quad k \in \mathbb{Z}
$$

exist and, when $\alpha$ is a finite positive Borel measure, that they satisfy either

$$
\text { (a) } \quad \sum_{n=1}^{\infty} c_{n}^{-\frac{1}{2 n}}=\infty \quad \text { or } \quad(b) \quad \sum_{n=1}^{\infty} c_{-n}^{-\frac{1}{2 n}}=\infty
$$

Let $\{k(n)\}$ be a sequence of nonnegative integers $0 \leq k(n) \leq 2 n$. In an earlier paper Gragg [8] (see also [10] and [11]) proved that when $k(n)=n$, the corresponding sequence of $2 \mathrm{PA}$ $\left\{F_{n}(z)\right\}$ converges to $F_{\alpha}(z)$ uniformly on $\mathbb{C}-[0, \infty)$. An alternative proof, making use of the theory of orthogonal Laurent polynomials was given in [7].

On the other hand, in several papers (see $[15,16,13]$ ), although in a more general context, López-Lagomasino proved the uniform convergence of sequences of 2 PAs to $F_{\alpha}(z)$ of the form $\left\{[k(n) / n]_{F_{\alpha}(z)}\right\}$ in compact sets of $\mathbb{C}-[0, \infty)$ by imposing either that $\lim _{n \rightarrow \infty}(2 n-$ $k(n))=\infty$ along with (5.2.a) or $\lim _{n \rightarrow \infty} k(n)=\infty$ along with (5.2.b). The papers by López-Lagomasino were initially inspired by Carleman's method [5]. Thus, in this section, we shall be first concerned with the uniform convergence of sequences of 2 PTAs to $F_{\alpha}(z), \alpha$ being in general a complex measure whose moments $c_{k}$ exist for all integer $k$. As usual in Padé-type approximation (see e.g. [2]), the key point is to make an appropriate choice for the denominators of the approximants. In our case we should choose it in order to achieve uniform convergence, preferably on the whole domain of analyticity of the function $F_{\alpha}$, i.e. on $\mathbb{C}-[0, \infty)$. In this respect, we have the following:

Theorem 5.1 Let $\alpha$ be a general measure such that the moments $c_{k}=\int_{0}^{\infty} x^{k} d \alpha(x)$ exist for all integer $k$. Assume $d \alpha(x)=\alpha^{\prime}(x) d x$ and consider an auxiliary finite positive Borel measure $d \sigma(x)=\sigma^{\prime}(x) d x$ satisfying (3.1) and whose moments $d_{k}=\int_{0}^{\infty} x^{k} d \sigma(x), k \in \mathbb{Z}$, satisfy either

$$
\lim _{n \rightarrow \infty} n-p(n)=\infty \quad \text { and } \quad \sum_{j=1}^{\infty} d_{j}^{-\frac{1}{2 j}}=\infty
$$

or

$$
\lim _{n \rightarrow \infty} p(n)=\infty \quad \text { and } \quad \sum_{j=1}^{\infty} d_{-j}^{-\frac{1}{2 j}}=\infty,
$$

$\{p(n)\}$ being a sequence of nonnegative integers with $0 \leq p(n) \leq n$. Let $Q_{n}^{\sigma}(x)$ be the nth orthogonal polynomial with respect to $d \sigma_{n}(x)=x^{-2 p(n)} d \sigma(x)$. Consider the 2PTA $(p(n) / n)_{F_{\alpha}}(z)$ whose denominator is $Q_{n}^{\sigma}(z)$. Then $\left\{(p(n) / n)_{F_{\alpha}}(z)\right\}_{n=1}^{\infty}$ converges uniformly to $F_{\alpha}(z)$ on any compact set of $\mathbb{C}-[0, \infty)$.

Proof. Set $\tilde{R}_{n}(z)=F_{\alpha}(z)-(p(n) / n)_{F_{\alpha}}(z)$. It is easily seen that

$$
\tilde{R}_{n}(z)=\frac{z^{p(n)}}{Q_{n}^{\sigma}(z)} \int_{0}^{\infty} \frac{Q_{n}^{\sigma}(x) d \alpha(x)}{x^{p(n)}(z-x)} .
$$

Take $z \in \mathbb{C}-[0, \infty)$. Assume first that $z$ is real, i.e. $z \in(-\infty, 0)$. Then, by the CauchySchwarz inequality, we can write

$$
\left|\tilde{R}_{n}(z)\right|^{2}=\left|\int_{0}^{\infty} \frac{z^{p(n)}}{Q_{n}^{\sigma}(z)} \frac{Q_{n}^{\sigma}(x)}{x^{p(n)}} \frac{\sqrt{\sigma^{\prime}(x)}}{\sqrt{x-t}} \frac{\alpha^{\prime}(x)}{\sqrt{\sigma^{\prime}(x)}} \frac{d x}{\sqrt{x-t}}\right|^{2}
$$




$$
\begin{aligned}
& \leq\left\{\int_{0}^{\infty}\left|\frac{z^{p(n)} Q_{n}^{\sigma}(x)}{Q_{n}^{\sigma}(z) x^{p(n)}} \sqrt{\frac{\sigma^{\prime}(x)}{x-z}}\right|^{2} d x\right\}\left\{\int_{0}^{\infty} \frac{\left|\alpha^{\prime}(x)\right|^{2}}{\sigma^{\prime}(x)} \frac{d x}{x-z}\right\} \\
& \leq M(z) K_{1}^{2} \int_{0}^{\infty}\left|\frac{z^{p(n)} Q_{n}^{\sigma}(x)}{Q_{n}^{\sigma}(z) x^{p(n)}} \sqrt{\frac{\sigma^{\prime}(x)}{x-z}}\right|^{2} d x \\
& =M(z) K_{1}^{2} \frac{z^{2 p(n)}}{\left(Q_{n}^{\sigma}(z)\right)^{2}} \int_{0}^{\infty} \frac{\left(Q_{n}^{\sigma}(x)\right)^{2} \sigma^{\prime}(x)}{x^{2 p(n)}(x-z)} d x \\
& =-M(z) K_{1}^{2}\left\{F_{\alpha}(z)-[2 p(n) / n]_{F_{\sigma}}(z)\right\} .
\end{aligned}
$$

Now, as it has been indicated, it follows from the assumptions (5.3)-(5.4) that the sequence $\left\{[2 p(n) / n]_{F_{\sigma}}(z)\right\}$ converges uniformly to $F_{\sigma}(z)$ on any compact subset of $\mathbb{C}-[0, \infty)$. Thus, from (5.5), the proof follows for $z \in(-\infty, 0)$.

Next, take $z \in \mathbb{C}-[0, \infty)$ and $z \notin \mathbb{R}$, i.e. $z \in \mathbb{C}-\mathbb{R}$. By applying again the CauchySchwarz inequality, one has

$$
\left|\tilde{R}_{n}(z)\right|^{2} \leq K_{1}^{2} \frac{\left|z^{2 p(n)}\right|}{\left|Q_{n}^{\sigma}(z)\right|^{2}} \int_{0}^{\infty} \frac{\left(Q_{n}^{\sigma}(x)\right)^{2}}{x^{2 p(n)}} \frac{\sigma^{\prime}(x)}{|z-x|^{2}} d x .
$$

On the other hand, set

$$
R_{n}^{\sigma}(z)=F_{\sigma}(z)-[2 p(n) / n]_{F_{\sigma}}(z)
$$

Thus we have (see [3])

$$
R_{n}^{\sigma}(z)=\frac{z^{2 p(n)}}{\left(Q_{n}^{\sigma}(z)\right)^{2}} \int_{0}^{\infty} \frac{\left(Q_{n}^{\sigma}(x)\right)^{2}}{x^{2 p(n)}} \frac{\sigma^{\prime}(x)}{(z-x)} d x .
$$

By the arguments given above, $R_{n}^{\sigma}(z)$ tends to zero uniformly on compact subsets of $\mathbb{C}-$ $[0, \infty)$. This implies that $\lim _{n \rightarrow \infty}\left|R_{n}^{\sigma}(z)\right|=0$ uniformly in $\mathbb{C}-[0, \infty)$. Now

$$
\begin{aligned}
\left|R_{n}^{\sigma}(z)\right| & =\frac{\left|z^{2 p(n)}\right|}{\left|Q_{n}^{\sigma}(z)\right|^{2}}\left|\int_{0}^{\infty} \frac{\left(Q_{n}^{\sigma}(x)\right)^{2}}{x^{2 p(n)}} \frac{\sigma^{\prime}(x)}{(z-x)} d x\right| \\
& =\frac{\left|z^{2 p(n)}\right|}{\left|Q_{n}^{\sigma}(z)\right|^{2}}\left|\int_{0}^{\infty} \frac{\left(Q_{n}^{\sigma}(x)\right)^{2}}{x^{2 p(n)}} \frac{(\bar{z}-x) \sigma^{\prime}(x)}{|z-x|^{2}}\right| \\
& =\left|\int_{0}^{\infty} \frac{\left|z^{2 p(n)}\right|}{\left|Q_{n}^{\sigma}(z)\right|^{2}} \frac{\left(Q_{n}^{\sigma}(x)\right)^{2}}{x^{2 p(n)}} \frac{(\bar{z}-x) \sigma^{\prime}(x)}{|z-x|^{2}} d x\right|=\left|H_{n}(z)\right| \rightarrow 0
\end{aligned}
$$

uniformly on compact subsets of $\mathbb{C}-[0, \infty)$. Since $\left|\operatorname{Re}\left(H_{n}(z)\right)\right| \leq\left|H_{n}(z)\right|$ and $\left|\operatorname{Im}\left(H_{n}(z)\right)\right| \leq$ $\left|H_{n}(z)\right|$, we deduce that

$$
\lim _{n \rightarrow \infty}\left|\operatorname{Re}\left(H_{n}(z)\right)\right|=\lim _{n \rightarrow \infty}\left|\operatorname{Im}\left(H_{n}(z)\right)\right|=0
$$

uniformly on compact subsets of $\mathbb{C}-[0, \infty)$.

Setting $z=\xi+i \eta$ and since $x \in \mathbb{R}(x \geq 0)$, we have

$$
\left|\operatorname{Im}\left(H_{n}(z)\right)\right|=|\eta| \frac{\left|z^{2 p(n)}\right|}{\left|Q_{n}^{\sigma}(z)\right|^{2} \mid} \int_{0}^{\infty} \frac{\left(Q_{n}^{\sigma}(x)\right)^{2}}{x^{2 p(n)}} \frac{\sigma^{\prime}(x)}{|z-x|^{2}} d x .
$$


By (5.7) and since $|\eta|>0$, we have that

$$
\lim _{n \rightarrow \infty} \frac{\left|z^{2 p(n)}\right|}{\left|Q_{n}^{\sigma}(z)\right|^{2}} \int_{0}^{\infty} \frac{\left(Q_{n}^{\sigma}(x)\right)^{2}}{x^{2 p(n)}} \frac{\sigma^{\prime}(x)}{|z-x|^{2}} d x=0
$$

uniformly on compact subsets of $\mathbb{C}-\mathbb{R}$. Thus, from (5.6) and (5.9), the proof follows.

Instead of condition (3.1) involving measures $\alpha$ and $\sigma$, we can impose a more restrictive one, but which is certainly easier to check. One has

Theorem 5.2 Under the same conditions as in Theorem 5.1, but now replacing (3.1) by

$$
\frac{\left|\alpha^{\prime}(x)\right|}{\sigma^{\prime}(x)} \leq K, \quad \forall x \in[0, \infty)
$$

the sequence $\left\{(p(n) / n)_{F_{\alpha}}\right\}_{n=1}^{\infty}$ of 2PTA to $F_{\alpha}(z)$ with as denominator $Q_{n}^{\sigma}(z), n=1,2, \ldots$ the nth orthogonalpolynomial with respect to $d \sigma_{n}(x)=x^{-2 p(n)} d \sigma(x)$, converges uniformly to $F_{\alpha}(z)$ on compact subsets of $\mathbb{C}-[0, \infty)$ when $\{p(n)\}_{1}^{\infty}$ is a sequence of nonnegative integers with $0 \leq p(n) \leq n$.

Proof. We have

$$
\tilde{E}_{n}(z)=F_{\alpha}(z)-(p(n) / n)_{F_{\alpha}}(z)=\frac{z^{p(n)}}{Q_{n}^{\sigma}(z)} \int_{0}^{\infty} \frac{Q_{n}^{\sigma}(x)}{z-x} \frac{\alpha^{\prime}(x)}{x^{p(n)}} d x .
$$

Thus

$$
\begin{aligned}
\left|\tilde{E}_{n}(z)\right| & \leq \frac{|z|^{p(n)}}{\left|Q_{n}^{\sigma}(z)\right|} \int_{0}^{\infty} \frac{\left|Q_{n}^{\sigma}(x)\right|}{|z-x|} \frac{\left|\alpha^{\prime}(x)\right|}{\sigma^{\prime}(x)} \frac{\sigma^{\prime}(x)}{x^{p(n)}} d x \\
& \leq K \frac{|z|^{p(n)}}{\left|Q_{n}^{\sigma}(z)\right|} \int_{0}^{\infty} \frac{\left|Q_{n}^{\sigma}(x)\right|}{|z-x|} \frac{\sigma^{\prime}(x)}{x^{p(n)}} d x .
\end{aligned}
$$

Hence by the Cauchy-Schwarz inequality, it follows that1

$$
\left|\tilde{E}_{n}(z)\right|^{2} \leq \frac{K^{2}|z|^{2 p(n)}}{\left|Q_{n}^{\sigma}(z)\right|^{2}}\left[\int_{0}^{\infty} \frac{\left|Q_{n}^{\sigma}(x)\right|^{2}}{|z-x|^{2}} \frac{\sigma^{\prime}(x)}{x^{2 p(n)}} d x\right]\left[\int_{0}^{\infty} \sigma^{\prime}(x) d x\right]
$$

where the last integral is the zeroth moment: $\int_{0}^{\infty} \sigma^{\prime}(x) d x=d_{0}$. Hence there exists a positive constant $M$ such that

$$
\left|\tilde{E}_{n}(z)\right|^{2} \leq M \frac{|z|^{2 p(n)}}{\left|Q_{n}^{\sigma}(z)\right|^{2}} \int_{0}^{\infty} \frac{\left|Q_{n}^{\sigma}(x)\right|^{2}}{|z-x|^{2}} \frac{\sigma^{\prime}(x)}{x^{2 p(n)}} d x .
$$

Let us consider the Cauchy transform $F_{\sigma}(z)$ of the measure $d \sigma$, and set

$$
E_{n}(z)=F_{\sigma}(z)-[p(n) / n]_{F_{\sigma}}(z) .
$$

We know that $\left|E_{n}(z)\right| \rightarrow 0$ as $n \rightarrow \infty$, uniformly on compact subsets of $\mathbb{C}-[0, \infty)$. Take first $z \in \mathbb{C}-[0, \infty), z=\xi+i \eta$ with $\eta \neq 0$, then

$$
E_{n}(z)=\frac{z^{2 p(n)}}{\left(Q_{n}^{\sigma}(z)\right)^{2}} \int_{0}^{\infty} \frac{\left(Q_{n}^{\sigma}(z)\right)^{2}}{|z-x|^{2}}(\bar{z}-x) \frac{\sigma^{\prime}(x)}{x^{2 p(n)}} d x
$$


and consequently

$$
\left|E_{n}(z)\right|=\frac{|z|^{2 p(n)}}{\left|Q_{n}^{\sigma}(z)\right|^{2}}\left|\int_{0}^{\infty} \frac{\left(Q_{n}^{\sigma}(x)\right)^{2}}{|z-x|^{2}}(\xi-x) \frac{\sigma^{\prime}(x)}{x^{2 p(n)}} d x-i \eta \int_{0}^{\infty} \frac{\left(Q_{n}^{\sigma}(x)\right)^{2}}{|z-x|^{2}} \frac{\sigma^{\prime}(x)}{x^{2 p(n)}} d x\right| .
$$

Taking the imaginary part, we get

$$
\frac{|z|^{2 p(n)}}{\left|Q_{n}^{\sigma}(z)\right|^{2}} \int_{0}^{\infty} \frac{\left(Q_{n}^{\sigma}(x)\right)^{2}}{|z-x|^{2}} \frac{\sigma^{\prime}(x)}{x^{2 p(n)}} d x \leq \frac{\left|E_{n}(z)\right|}{|\eta|}
$$

So, by (5.11) and (5.12), we see that $E_{n}(z) \rightarrow 0$ uniformly on compact subsets of $\mathbb{C}-\mathbb{R}$.

It remains to consider $z \in \mathbb{R}, z<0$. This case is more immediate. Indeed,

$$
\left|\tilde{E}_{n}(z)\right| \leq \frac{|z|^{p(n)}}{\left|Q_{n}^{\sigma}(z)\right|} K \int_{0}^{\infty} \frac{\left|Q_{n}^{\sigma}(x)\right|}{(x-z)} \frac{\sigma^{\prime}(x)}{x^{p(n)}} d x
$$

which gives

$$
\left|\tilde{E}_{n}(z)\right| \leq K^{2}\left|E_{n}(z)\right| \int_{0}^{\infty} \frac{\sigma^{\prime}(x)}{x-z} d x
$$

and the proof is complete.

Let us now study the convergence of the sequence of quadrature formulas

$$
I_{n}(f)=\sum_{j=1}^{n} \tilde{A}_{j, n} f\left(x_{j, n}\right)
$$

where $\left\{x_{j, n}\right\}$ are the zeros of $Q_{n}^{\sigma}(x)$, the $n$th orthogonal polynomial with respect to $x^{-2 p(n)} d \sigma(x)$, with $\sigma$ an auxiliary measure as given in Theorem 5.1, so that

$$
I_{n}(f)=I_{\alpha}(f), \quad \forall f \in \Lambda_{-p(n), n-1-p(n)} .
$$

We are still assuming that $\alpha$ is a general measure whose moments $c_{k}$ exist for all integer $k$ and (3.1) holds. Let us denote by $C^{B}[0, \infty)$ the class of continuous functions $f$ defined on $[0, \infty)$ such that $\lim _{x \rightarrow+\infty} f(x)$ exists and is a finite number (real or complex). The following holds:

Theorem 5.3 Under the conditions above, one has

$$
\lim _{n \rightarrow \infty} I_{n}(f)=I_{\alpha}(f), \quad \forall f \in C^{B}[0, \infty),
$$

if and only if there exists a positive constant $M$ such that

$$
\sum_{j=0}^{n}\left|\tilde{A}_{j, n}\right| \leq M \quad \text { for } n=1,2, \ldots
$$

Proof. Let us first consider the corresponding sequence of 2PTAs $(p(n) / n)_{F_{\alpha}}(z)$ with denominator $Q_{n}^{\sigma}(x)$ (supposed to be monic), one knows that

$$
(p(n) / n)_{F_{\alpha}}(z)=\sum_{j=1}^{n} \frac{\tilde{A}_{j, n}}{z-x_{j, n}} .
$$


Let $\left\{z_{j}\right\}_{j=1}^{n}$ be a sequence of complex numbers in $\mathbb{C}-[0, \infty)$ satisfying the condition $z_{j} \neq z_{k}$ for $j \neq k$ and

$$
\sum_{\operatorname{Im}\left(\sqrt{z_{j}}\right)>0} \frac{\operatorname{Im}\left(\sqrt{z_{j}}\right)}{1+\left|z_{j}\right|}=+\infty
$$

By [1], we know that the family $\left\{1,1 /\left(z-z_{1}\right), \ldots, 1 /\left(z-z_{j}\right), \ldots\right\}$ is dense in the class $C^{B}[0, \infty)$ with respect to the uniform norm. Clearly

$$
I_{n}\left(\frac{1}{x-z_{k}}\right)=-(p(n) / n)_{F_{\alpha}}\left(z_{k}\right)
$$

and

$$
I_{\alpha}\left(\frac{1}{x-z_{k}}\right)=-F_{\alpha}\left(z_{k}\right)
$$

Thus, by Theorem 5.1, it follows that

$$
\lim _{n \rightarrow \infty} I_{n}\left(\frac{1}{x-z_{k}}\right)=I_{\alpha}\left(\frac{1}{x-z_{k}}\right) \quad k=1,2, \ldots
$$

Thus, under these conditions, the proof is a direct consequence of the Banach-Steinhaus Theorem [12].

As for the Gaussian quadrature formulas, we have

Corollary 5.4 Let $\alpha$ be a finite positive Borel measure on $[0, \infty)$ such that the moments $\left\{c_{k}\right\}_{-\infty}^{\infty}$ satisfy either

$$
\lim _{n \rightarrow \infty} 2 n-k(n)=\infty \quad \text { and } \quad \sum_{j=1}^{\infty} c_{j}^{-\frac{1}{2 j}}=\infty
$$

or

$$
\lim _{n \rightarrow \infty} k(n)=\infty \quad \text { and } \quad \sum_{j=1}^{\infty} c_{j}^{-\frac{1}{2 j}}=\infty
$$

with $\{k(n)\}$ a sequence of integers with $0 \leq k(n) \leq 2 n$. Let $I_{n}(f)=\sum_{j=1}^{n} A_{j, n} f\left(x_{j, n}\right)$ be the $n$-point Gaussian quadrature formula in $\Lambda_{-k(n), 2 n-k(n)-1}$. Then $\lim _{n \rightarrow \infty} I_{n}(f)=I_{\alpha}(f)$ for all functions $f \in C^{B}[0, \infty)$.

Proof. Since $\alpha$ is now a positive measure, we can choose as auxiliary measure $d \sigma(x)=d \alpha(x)$. Thus the $n$-point interpolatory quadrature formula becomes a Gaussian quadrature formula. Furthermore,

$$
\int_{0}^{\infty} \frac{\left|\alpha^{\prime}(x)\right|^{2}}{\sigma^{\prime}(x)} d x=\int_{0}^{\infty} d \alpha(x)=c_{0}<\infty
$$

and (3.1) is trivially satisfied. On the other hand, and as was already mentioned in the beginning of this section, the sequence of 2 PAs $[k(n) / n]_{F_{\alpha}}(z)$ converges to $F_{\alpha}(z)$ for all $z \in \mathbb{C}-[0, \infty)$ by $(5.13)-(5.14)$. Finally, since $A_{j, n}>0$ for $j=1,2, \ldots, n$ and $n=1,2, \ldots$ we have

$$
\sum_{j=1}^{n}\left|A_{j, n}\right|=\sum_{j=1}^{n} A_{j, n}=\int_{0}^{\infty} d \alpha(x)=c_{0}<\infty
$$

and the corollary follows. 
Remark 5.1 Of course Theorem 5.3 and Corollary 5.4 also hold when (3.1) is replaced by (5.10).

In the rest of this section we will deduce estimates for the rate of convergence of these quadrature formulas, both of interpolatory type and of Gaussian type. In this respect, it is convenient to recall that in a recent paper, López-Lagomasino and Martinez-Finkelshtein gave exact rates of convergence for any sequence of $2 \mathrm{PAs}\left\{[k(n) / n]_{F_{\alpha}}(z)\right\}$ to $F_{\alpha}(z)$ under the assumption that

$$
\lim _{n \rightarrow \infty} \frac{k(n)}{2 n}=\theta \in[0,1]
$$

$\alpha$ being a measure with special features. More precisely, $d \alpha$ is given by

$$
d \alpha(x)=x^{\nu} \exp (-\tau(x)) d x
$$

where $\nu \in \mathbb{R}$ and $\tau(x)$ is a continuous function on $(0, \infty)$ that satisfies for $\gamma>1 / 2$ and $s>0$

$$
\lim _{x \rightarrow 0^{+}}(s x)^{\gamma} \tau(x)=\lim _{x \rightarrow+\infty}(s x)^{-\gamma} \tau(x)=1 .
$$

Those authors prove in [14] the following fundamental result

Theorem 5.5 Let $\left\{Q_{n}^{\alpha}(x)\right\}$ denote the sequence of orthonormal polynomials with respect to the varying measure $d \alpha_{n}(x)=x^{-k(n)} d \alpha(x), n=1,2, \ldots$. Then, uniformly on compact sets in $\mathbb{C}-\mathbb{R}$, one has

$$
\lim _{n \rightarrow \infty} \frac{\log \left|Q_{n}^{\alpha}(z) / z^{k(n) / 2}\right|}{(2 n)^{r}}=D(\gamma) \delta(z)
$$

where

$$
D(\gamma)=\frac{2 \gamma}{2 \gamma-1}\left[\frac{\Gamma(\gamma+1 / 2)}{\sqrt{\pi} \Gamma(\gamma)}\right]^{\frac{1}{2 \gamma}}
$$

$\Gamma$ is the Euler Gamma function, and

$$
\delta(z)=(1-\theta)^{r} \operatorname{Im}\left((s z)^{1 / 2}\right)+\theta^{r} \operatorname{Im}\left((s z)^{-1 / 2}\right)
$$

with $r=1-1 /(2 \gamma)<1$. The branch of the root is taken such that $(-1)^{1 / 2}=i$.

Also the following result about the exact rate of convergence for a general sequence of $2 \mathrm{PA}$ is given in the same paper: Set $R_{n}(z)=F_{\alpha}(z)-[k(n) / n]_{F_{\alpha}}(z)$ and define for any compact set $K \subset \mathbb{C}-[0, \infty)$ the norm $\left\|R_{n}\right\|_{K}=\sup _{z \in K}\left|R_{n}(z)\right|$, then

$$
\lim _{n \rightarrow \infty} \frac{\log \left\|R_{n}\right\|_{K}}{(2 n)^{r}}=-2 D(\gamma) \inf _{z \in K}\{\delta(z)\} .
$$

Example 5.1 As an example, let us consider the one which was also considerd in [10] where

$$
d \alpha(x)=\exp \left(-x-x^{-1}\right), \quad x \in(0, \infty)
$$

and $k(n)=n$. Here $\tau(x)=\left(x^{2}+1\right) / x, \nu=0$ and $s=\gamma=1$. Furthermore $\theta=1 / 2, r=1 / 2$ and $D(\gamma)=D(1)=\sqrt{2}$. Take the compact set $K=[-b,-a]$ with $-\infty<-b<-1<-a<$ 0 . In this case (5.17) becomes

$$
\lim _{n \rightarrow \infty}\left\|R_{n}\right\|_{[-b,-a]}^{1 / \sqrt{n}}=e^{-4} .
$$

If we consider Padé approximants at $\infty$, i.e., $k(n)=0$ for all $n$, then $\theta=0$ and the right-hand side of (5.18) has to be replaced by

$$
e^{-2 \sqrt{2 a}}>e^{-4}
$$


From (5.17), one can derive the convergence of the sequence of Gaussian quadrature formulas in $\Lambda_{-k(n), 2 n-1-k(n)}$ for the class of analytic functions. Indeed, one has

Theorem 5.6 Under the same conditions as in Theorem 3.1, let $I_{n}(f)$ be the n-point Gaussian formula in $\Lambda_{-k(n), 2 n-1-k(n)}$, and let $f$ be an analytic function in a domain $V$ such that $[0, \infty) \subset V \subset \hat{\mathbb{C}}$. Then

$$
\lim _{n \rightarrow \infty} I_{n}(f)=I_{\alpha}(f):=\int_{0}^{\infty} f(x) d \alpha(x)
$$

Furthermore

$$
\limsup _{n \rightarrow \infty}\left|I_{\alpha}(f)-I_{n}(f)\right|^{1 /(2 n)^{r}} \leq \lambda<1
$$

where $\lambda=\exp (-R)$ and

$$
R=2 D(\gamma) \inf _{z \in \Gamma}\{\delta(z)\}>0
$$

with $r, \delta(z)$, and $D(\gamma)$ as given in Theorem 5.5, and $\Gamma$ a Jordan curve contained in $V$.

Proof. Since $\Gamma$ is a Jordan curve in $V$, we can use the Cauchy and Fubini Theorem to get

$$
I_{\alpha}(f)-I_{n}(f)=\int_{\Gamma} f(z)\left[F_{\alpha}(z)-F_{n}(z)\right] d z
$$

where $F_{n}(z)=[k(n) / n]_{F_{\alpha}}(z)$ is the $2 \mathrm{PA}$ of $F_{\alpha}$. Thus there exists a positive constant $K=$ $K(\Gamma)$ so that

$$
\left|I_{\alpha}(f)-I_{n}(f)\right| \leq K(\Gamma)\left\|R_{n}\right\|_{\Gamma}
$$

with $\left\|R_{n}\right\|_{\Gamma}=\sup _{z \in \Gamma}\left|F_{\alpha}(z)-F_{n}(z)\right|$. Now by (5.17) the proof immediately follows.

Let us next assume that $\alpha$ is a general (possibly complex) measure such that the moments $c_{k}$ of (5.1) exist. Assume $d \alpha(x)=\alpha^{\prime}(x) d x$ and consider an auxiliary finite Borel measure $d \sigma(x)=\sigma^{\prime}(x) d x$ of the type (5.15-5.16), where $\sigma^{\prime}$ satisfies the conditions given in Theorem 3.1. Let $\{p(n)\}$ be a sequence of nonnegative integers such that

$$
\lim _{n \rightarrow \infty} \frac{p(n)}{n}=\theta \in[0,1], \quad \text { and } \quad 0 \leq p(n) \leq n .
$$

Let $Q_{n}^{\sigma}(x)$ be the $n$-th orthonormal polynomial with respect to $d \sigma_{n}(x)=x^{-2 p(n)} d \sigma(x)$. Consider the 2PTA $(p(n) / n)_{F_{\alpha}}(x)$ whose denominator is $Q_{n}^{\sigma}(x)$. Then we have

Theorem 5.7 Under the conditions just introduced, the 2PTA $(p(n) / n)_{F_{\alpha}}(x)$ converge uniformly to $F_{\alpha}(z)$ on any compact set $K \subset \mathbb{C}-[0, \infty)$ as $n \rightarrow \infty$.

Furthermore we have for the error $\tilde{R}_{n}=F_{\alpha}-(p(n) / n)_{F_{\alpha}}$

$$
\limsup _{n \rightarrow \infty}\left\|\tilde{R}_{n}\right\|_{K}^{1 /(2 n)^{r}} \leq \sqrt{\lambda}<1
$$

with $r$ and $\lambda$ as in Theorem 5.6.

Proof. It is easily seen that

$$
\tilde{R}_{n}(z)=\frac{z^{p(n)}}{Q_{n}^{\sigma}(z)} \int_{0}^{\infty} \frac{Q_{n}^{\sigma}(x) d \alpha(x)}{x^{p(n)}(x-z)}
$$


Thus, from (3.1) and the Cauchy-Schwarz inequality, it follows that there exists a positive constant $M=M(K)$ such that

$$
\left|\tilde{R}_{n}(z)\right| \leq \frac{|z|^{p(n)}}{\left|Q_{n}^{\sigma}(z)\right|} M, \quad \forall z \in K \subset \mathbb{C}-[0, \infty)
$$

Now take $k(n)=2 p(n)$, then

$$
\lim _{n \rightarrow \infty} \frac{k(n)}{2 n}=\lim _{n \rightarrow \infty} \frac{p(n)}{n}=\theta \in[0,1] .
$$

As before, consider $r=1-1 /(2 \gamma)$, then

$$
\limsup _{n \rightarrow \infty}\left\|\tilde{R}_{n}\right\|_{K}^{1 /(2 n)^{r}} \leq \sqrt{\lambda}
$$

with $\lambda<1$ as in Theorem 5.6, and the proof thus follows.

With the zeros of $Q_{n}^{\sigma}$, we can construct an $n$-point interpolatory quadrature formula exact in $\Lambda_{-p(n), n-1-p(n)}$, say $\tilde{I}_{n}(f)=\sum_{j=1}^{n} \tilde{A}_{j} f\left(x_{j}\right)$. Let $f$ be an analytic function in an open set $V$ of $\hat{\mathbb{C}}$ such that $[0, \infty) \subset V$. Then we have

Theorem 5.8 Under the same conditions as in the previous theorem and with the interpolatory quadrature formula $\tilde{I}_{n}$ as defined above we have

$$
\lim _{n \rightarrow \infty} \tilde{I}_{n}(f)=I_{\alpha}(f):=\int_{0}^{\infty} f(x) d \alpha(x) .
$$

Furthermore

$$
\limsup _{n \rightarrow \infty}\left|I_{\alpha}(f)-\tilde{I}_{n}(f)\right|^{1 / n^{r}} \leq \sqrt{\lambda}<1
$$

whith $r$ and $\lambda$ as in Theorem 5.6.

Proof. Like in Theorem 5.6, it holds that

$$
I_{\alpha}(f)-\tilde{I}_{n}(f)=\int_{\Gamma} f(z)\left[F_{\alpha}(z)-(p(n) / n)_{F_{\alpha}}(z)\right] d z .
$$

Thus, if $\Gamma$ is a Jordan curve contained in $V$, there exists a positive constant $M=M(\Gamma)$ such that

$$
\left|I_{\alpha}(f)-\tilde{I}_{n}(f)\right| \leq M(\Gamma)\left\|F_{\alpha}-(p(n) / n)_{F_{\alpha}}\right\|_{\Gamma} .
$$

Now the proof follows from (5.20).

Example 5.2 Let us take again $d \sigma(x)=d \alpha(x)=\exp \left(-x-x^{-1}\right), x \in[0, \infty)$. Thus $\nu=0, s=\gamma=1, r=1 / 2$, and $D(\gamma)=D(1)=\sqrt{2}$. Let us consider the integrand $f(z)=\exp \left\{(z+a)^{-1}\right\}, a>0$ which represents an analytic function in $\hat{\mathbb{C}}$ except in the point $z=-a$, where it has an essential singularity. For the Jordan curve $\Gamma$, we take

$$
\Gamma_{\epsilon, a}=\{z:|z+a|=\epsilon\}
$$


with $\epsilon$ as small as possible. In order to compare the estimations of the rate of convergence both for classical Gaussian quadrature formulas $(k(n)=0$ for all $n)$, and Gaussian formulas in $\Lambda_{-k(n), 2 n-1-k(n)}$ (say we choose $k(n)=n$ for all $n$ ), we have to compute

$$
\delta_{1}=\inf _{z \in \Gamma_{\epsilon, a}}\{\operatorname{Im}(\sqrt{z})\}, \quad(\text { classical Gaussian, } \theta=0)
$$

and

$$
\delta_{2}=\inf _{z \in \Gamma_{\epsilon, a}}\{\operatorname{Im}(\sqrt{z})+\operatorname{Im}(\sqrt{1 / z})\}, \quad(\theta=1 / 2) .
$$

Thus the estimates for the rates of convergence for the corresponding quadrature formules are given by

$$
\lambda_{1}=\exp \left\{-2 \sqrt{2} \delta_{1}\right\}, \quad(\theta=0)
$$

and

$$
\lambda_{2}=\exp \left\{-2 \delta_{2}\right\}, \quad(\theta=1 / 2) .
$$

\section{References}

[1] N.I. Achieser. Theory of approximation. Frederick Ungar Publ. Co., New York, 1956.

[2] C. Brezinski. Padé-type approximation and general orthogonal polynomials, volume 50 of Internat. Ser. of Numer. Math. Birkhäuser Verlag, Basel, 1980.

[3] A. Bultheel, C. Díaz-Mendoza, P. González-Vera, and R. Orive. Quadrature on the half line and two-point Padé approximants to Stieltjes functions. Part II: Convergence. $J$. Comput. Appl. Math., 77:53-76, 1997.

[4] A. Bultheel, P. González-Vera, and R. Orive. Quadrature on the half line and two-point Padé approximants to Stieltjes functions. Part I: Algebraic aspects. J. Comput. Appl. Math., 65:57-72, 1996.

[5] T. Carleman. Les fonctions quasi analytiques. Gauthier-Villars, Paris, 1926.

[6] A. Draux. Polynômes orthogonaux formels - applications, volume 974 of Lecture Notes in Math. Springer, Berlin, 1983.

[7] P. González-Vera and $\mathrm{O}$. Njåstad. Convergence of two-point Padé approximants to series of Stieltjes. J. Comput. Appl. Math., 32:97-105, 1990.

[8] W.B. Gragg. Truncation error bounds for T-fractions. In E.W. Cheney, editor, Approximation Theory III, pages 455-460, New York, 1980. Academic Press.

[9] W.B. Jones, O. Njåstad, and W.J. Thron. Two-point Padé expansions for a family of analytic functions. J. Comput. Appl. Math., 9:105-124, 1983.

[10] W.B. Jones and W.J. Thron. Survey of continued fraction methods of solving moment problems and related topics. In W.B. Jones, W.J. Thron, and H. Waadeland, editors, Analytic Theory of Continued Fractions, volume 932 of Lecture Notes in Math., pages 4-37, Berlin, 1982. Springer. 
[11] W.B. Jones, W.J. Thron, and H. Waadeland. A strong Stieltjes moment problem. Trans. Amer. Math. Soc., 206:503-528, 1980.

[12] V.J. Krylov. Approximate calculation of integrals. MacMillan, 1962.

[13] G. López. Convergence of Padé approximants of Stieltjes type meromorphic functions and comparative asymptotice for orthogonal polynomials. Math. USSR-Sb., 64:207-227, 1989 .

[14] G. López-Lagomasino and A.M. Finkelshtein. Rate of convergence of two-point Padé approximants and logarithmic asymptotics of Laurent-type orthogonal polynomials. Constr. Approx., 11:255-286, 1995.

[15] G.L. Lopes [López-Lagomasino]. On the convergence of Padé approximants for Stieltjes type functions. Math. USSR-Sb., 39:281-288, 1981.

[16] G.L. Lopes [López-Lagomasino]. On the asymptotics of the ratio of orthogonal polynomials and convergence of multipoint Padé approximants. Math. USSR-Sb., 56:207-219, 1985 .

[17] J. Stoer and R. Bulirsch. Introduction to numerical analysis. Springer, 1983. 